\title{
Prevalence of Potentially Malignant Lesions and Oral Cancer Among Smokers in an Egyptian cohort: A Hospital-based Cross-Sectional Study
}

\author{
Amira M. Abd el-Aziz ${ }^{1}$, Alaa AbouShousha ${ }^{1}$, Shereen Ali ${ }^{1}$, Fat'heya Zahran ${ }^{1}$ \\ ${ }^{1}$ Oral Medicine and Periodontology Department, Faculty of Dentistry, Cairo University, Egypt.
}

Email: amiramohamedd92@gmail.com

Submitted: 8-3-2020

Accepted: 1-6-2020

\begin{abstract}
Objectives: This cross-sectional study was designed to determine the prevalence of potentially malignant lesions and oral cancer among smokers.

Methods: The current observational cross-sectional study was conducted in the Clinics of Oral Medicine and Periodontology department and the Diagnostic center, Faculty of Dentistry - Cairo University in Cairo- Egypt. Smokers from all age groups and both genders who agreed to sign the informed consent were included in the study. Detailed history of tobacco consumption, different forms of tobacco use, drugs, alcohol consumption and quitting trials were recorded and followed by clinical examination and histopathological assessment of suspicious lesions.

Results: Among the 1664 enrolled participants, 3.54\% had leukoplakia, 2.88\% had oral lichen planus, $1.5 \%$ had oral squamous cell carcinoma and $0.12 \%$ had verrucous carcinoma.

Conclusion: leukoplakia was the most frequently encountered lesion followed by oral lichen planus. Dentists must be aware of the risk of smoking on oral mucosa and the early signs of developing premalignant or malignant lesions.
\end{abstract}

Keywords: prevalence, smokers, potentially malignant lesions, leukoplakia, oral cancer

\section{Introduction}

The oral cavity of an individual is exposed to numerous irritants through its lifetime. The oral mucosa serves as a protective barrier against trauma, pathogens, and carcinogenic irritants (Ali et al., 2013). Among the harmful irritants, tobacco is considered to have the most detrimental impact on oral health (Sham et al., 2003).

Studies have shown that tobacco smoking is responsible for many potentially malignant and malignant lesions including: smoker's keratosis, leukoplakia and oral squamous cell carcinoma
(OSCC) (Kuper et al., 2002; Taybos, 2003; Scully and Felix, 2005; Vellappally et al., 2007 and Motamayel et al., 2013 and Behura et al., 2015).

In Egypt, $30 \%$ of adults are current smokers. smoking prevalence is much less among females $(0.6 \%)$ than males $(38.1 \%)$. Almost $96 \%$ of men, who use tobacco, do so daily. Men are more likely to use manufactured cigarettes $(31.8 \%)$ than shisha $(6.2 \%)$ or smokeless tobacco $(4.1 \%)$ Few women use tobacco (cigarettes $(0.2 \%)$, shisha $(0.3 \%)$ and smokeless tobacco $(0.3 \%)$; however, all women who currently smoke shisha do so daily (Fouda et al., 2018). 
Despite all the known hazards of smoking and its association with potentially malignant lesions and oral cancer, there is a deficiency in the data concerned with the prevalence of these lesions among smokers in Egypt. To further understand the relation between smoking and oral carcinogenesis, this crosssectional study was designed to determine the prevalence of potentially malignant lesions and oral cancer among smokers in an Egyptian cohort who attended to the clinics of Oral Medicine and Periodontology department and the Diagnostic center, Faculty of Dentistry - Cairo University.

\section{Methodology}

The current observational cross-sectional study was conducted in the Clinics of Oral Medicine and Periodontology department and the Diagnostic center, Faculty of Dentistry - Cairo University from April 2017 to April 2019. The study was approved by the Research ethics committee of Faculty of Dentistry, Cairo University.

Smokers from all age groups and both genders who agreed to sign the informed consent were included in the study. Individuals suffering from any systemic disease or taking medications as well as non-Egyptians were excluded.

For each eligible participant, a full history including education level, socioeconomic level history of tobacco smoking was obtained and different forms of tobacco use were noted with options include shisha, cigars or pipes. Age of first starting to consume tobacco, duration of smoking, daily or less than daily smoker, dose of tobacco per day and history of smoking more than 4 packs of cigarettes per day were also recorded.

Smokers were classified into past smoker (P), current smoker (C), smoker (S), non-smoker (N), and unknown (U). Past smokers are patients who have not smoked for at least one year. Current smokers include patients who give history indicating that they are smokers at the time being examined or have smoked within the past year. Smokers were considered light smoker ( $\leq 10$ cigarette equivalent), moderate (11-20 cigarette equivalent), and heavy smoker (>20 cigarette equivalent). The different types of tobacco use were assessed according to (WHO 2013) as each shisha session lasts 80-20 minutes that is equal to 100 cigarettes.

The history of consuming alcohol or illegal drugs was recorded including the duration, frequency and addiction to such habits. Any quitting trials were recorded with the duration and method of quitting.

A thorough clinical examination was conducted for the whole oral cavity. Detected lesions was diagnosed by assessment of the findings of the history, clinical examination and histopathological evaluation. All participants were educated about the hazardous effects of smoking, its adverse effects on both general and oral health. They were all advised strongly to quit this habit.

The primary outcome was identifying and recording potentially malignant lesions and oral cancer which includes "oral lichen planus, leukoplakia, OSCC and verrucous carcinoma" following the diagnostic criteria of (Scully and Felix, 2005 and Behura et al., 2015).

The secondary outcome was histopathological evaluation to detect dysplastic and malignant changes using (Warnakulasuriy et al., 2007 and Rastogi et al., 2013) in which zero equals No dysplasia, 1 equals Mild dysplasia: Increased number of cells in the basal and parabasal epithelial regions showing nuclear hyperchromatic and pleomorphism, 2 equals Moderate dysplasia: Bulbous rete-pegs with increased numbers cells showing nuclear hyperchromatism and pleomorphism, extending to and including the basal, parabasal and prickle cell layer, 3 equals Severe dysplasia: Bulbous retepegs with increased numbers of cells showing nuclear hyperchromatism and pleomorphism through the entire thickness of epithelium and 4 equals Carcinoma in situ: Markedly atypical changes showing nuclear hyperchromatism and pleomorphism and encompassing the entire thickness of the epithelium.

Sample size calculation was based on a previous paper by Patil et al. (2013), where the prevalence of oral mucosal lesions among smokers was $26.8 \%$. Using a precision of 3 , a design effect set at 2 with 
95\% CI (confidence interval), a total sample size of 1664 was found to be sufficient. The sample size was calculated by Epi info.

Categorical data was presented as frequencies and percentages and was analyzed using chi square test. Correlation was performed in terms of Spearman's Rho (rs) correlation. The significance level was set at $\mathrm{P} \leq 0.05$. Statistical analysis was performed with IBM® SPSS® Statistics Version 25 for Windows.

\section{Results}

The participants demographic data and smoking frequency is shown in (Table 1). The education level ranges from $14.6 \%$ had no education, $55.2 \%$ basic education and only $30 \%$ received high education. City residence reached $60.27 \%$

Cigarettes were the commonest type of tobacco smoked $(82.1 \%)$ followed by mixed cigarettes and shisha (11.2\%) and the least was shisha (6.6\%). No subjects used pipes or cigars. As was considered $15.08 \%$ of the patients were light smokers, $37.92 \%$ were moderate smokers and about $47 \%$ were heavy smokers (Table 1).

Table (1): Demographic data and characteristics of the participants

\begin{tabular}{|c|c|c|c|}
\hline & & $\bar{n}$ & $\%$ \\
\hline \multirow[t]{2}{*}{ Gender } & Male & 1624 & $97.6 \%$ \\
\hline & Female & 40 & $2.4 \%$ \\
\hline \multirow[t]{6}{*}{ Age (years) } & $\leq 20$ & 48 & $2.88 \%$ \\
\hline & $21-30$ & 212 & $12.74 \%$ \\
\hline & $31-40$ & 421 & $25.3 \%$ \\
\hline & $41-50$ & 375 & $22.53 \%$ \\
\hline & $51-60$ & 302 & $18.14 \%$ \\
\hline & $\geq 60$ & 306 & $18.39 \%$ \\
\hline \multirow{6}{*}{$\begin{array}{c}\text { Duration of smoking } \\
\text { (years) }\end{array}$} & $\leq 10$ & 460 & $27.64 \%$ \\
\hline & $11-20$ & 470 & $28.24 \%$ \\
\hline & $21-30$ & 431 & $25.9 \%$ \\
\hline & $31-40$ & 158 & $9.49 \%$ \\
\hline & $41-50$ & 131 & $7.87 \%$ \\
\hline & $\geq 51$ & 14 & $0.84 \%$ \\
\hline \multirow{6}{*}{$\begin{array}{l}\text { Dose of smoking } \\
\text { (cigarette/day) }\end{array}$} & $\leq 10$ & 251 & $15.08 \%$ \\
\hline & $11-20$ & 631 & $37.92 \%$ \\
\hline & $21-30$ & 256 & $15.38 \%$ \\
\hline & $31-40$ & 401 & $24.09 \%$ \\
\hline & $41-50$ & 77 & $4.62 \%$ \\
\hline & $\geq 51$ & 48 & $2.88 \%$ \\
\hline \multirow[t]{3}{*}{ Education level } & No Education & 244 & $14.6 \%$ \\
\hline & Basic education & 920 & $55.2 \%$ \\
\hline & High education & 500 & $30 \%$ \\
\hline \multirow[t]{2}{*}{ Residence } & City & 1003 & $\overline{60.27 \%}$ \\
\hline & Countryside & 661 & $39.7 \%$ \\
\hline \multirow{4}{*}{$\begin{array}{l}\text { Drug addiction \& Alcohol } \\
\text { consumption }\end{array}$} & No & 1190 & $71.5 \%$ \\
\hline & Hashish & 228 & $13.7 \%$ \\
\hline & Alcohol & 136 & $8.17 \%$ \\
\hline & Other drugs & 110 & $6.6 \%$ \\
\hline \multirow[t]{2}{*}{ Quitting trials } & No & 1552 & $93.26 \%$ \\
\hline & Yes & 112 & $6.73 \%$ \\
\hline
\end{tabular}


Majority of the participant didn't report quitting trials $(93.26 \%)$ only $(6.73 \%)$ did (Table 1). Among those who tried to quite tobacco consumption 4 cases showed leukoplakia with no dysplasia.

Among the recruited participants, 59 participants (3.54\%) had leukoplakia, 48 participants $(2.88 \%)$ had oral lichen planus (OLP), 25 participants (1.5\%) had OSCC and only 2 participants $(0.12 \%)$ had verrucous carcinoma. Histopathological evaluation showed that 10 lesions of the 59 leukoplakia cases were dysplastic and 2 lesions of the 48 OLP cases were dysplastic (Table 2).

Leukoplakia presented on different oral mucosal sites including commissures of both sides, buccal mucosa, floor of the mouth and lateral border of the tongue respectively while oral lichen planus was mainly on buccal mucosa and dorsum surface of the tongue.

Patients with OSCC showed lower level of education ranging from no education to basic education and mostly were country side residence. 2 cases of 25 OSCC reported consuming cigarettes and shisha together.

The total number of lesions presence in association with each duration of smoking is presented in (Table 3). The correlation between lesion presence and duration of smoking was statistically insignificant. However, there was a trend of increase in lesion presence by time in relation to number of patients in each duration of smoking.

Table (2): Prevalence of potentially malignant lesions and oral cancer

\begin{tabular}{lccccc}
\hline \multicolumn{1}{c}{ Lesions } & $\mathbf{N}$ & \% & \multicolumn{3}{c}{ Histopathological assessment } \\
\cline { 4 - 6 } & & & No & Dysplasia & SCC \\
\hline Leukoplakia & 59 & 3.54 & 49 & 10 & 0 \\
\hline Oral lichen planus & 48 & 2.88 & 46 & 2 & 0 \\
\hline Oral squamous cell carcinoma & 25 & 1.5 & - & - & 25 \\
\hline Verrucous Carcinoma & 2 & 0.12 & - & - & 2 \\
\hline
\end{tabular}

Table (3): Prevalence of oral lesions regarding duration of smoking

\begin{tabular}{|c|c|c|c|c|c|c|c|c|}
\hline \multirow[t]{2}{*}{ Lesions } & \multicolumn{6}{|c|}{ Duration of smoking (y) } & \multirow{2}{*}{$\operatorname{Rho}\left(r_{s}\right)$} & \multirow{2}{*}{ P-value } \\
\hline & $\leq \mathbf{1 0}$ & 11-20 & 21-30 & $31-40$ & 41-50 & $\geq 51$ & & \\
\hline \multirow[t]{2}{*}{ Leukoplakia (n; \%) } & 3 & 13 & 26 & 5 & 12 & 0 & \multirow{2}{*}{-0.3142} & \multirow{2}{*}{0.5638} \\
\hline & $0.65 \%$ & $2.76 \%$ & $6 \%$ & $3.16 \%$ & $9.16 \%$ & $0.0 \%$ & & \\
\hline \multirow[t]{2}{*}{ Oral lichen planus (n; \%) } & 11 & 10 & 15 & 3 & 8 & 1 & \multirow{2}{*}{-0.7714} & \multirow{2}{*}{0.1028} \\
\hline & $2.4 \%$ & $2.12 \%$ & $3.5 \%$ & $1.9 \%$ & $6.1 \%$ & $7.14 \%$ & & \\
\hline \multirow[t]{2}{*}{$\operatorname{OSCC}(\mathbf{n} ; \%)$} & 1 & 6 & 8 & 5 & 4 & 1 & \multirow{2}{*}{-0.2319} & \multirow{2}{*}{0.6583} \\
\hline & $0.2 \%$ & $1.27 \%$ & $1.85 \%$ & $3.16 \%$ & $3 \%$ & $7.14 \%$ & & \\
\hline \multirow[t]{2}{*}{ Verrucous Carcinoma (n; \%) } & 0 & 0 & 1 & 0 & 1 & 0 & \multirow{2}{*}{0.2070} & \multirow{2}{*}{0.6939} \\
\hline & $0.0 \%$ & $0.0 \%$ & $0.23 \%$ & $0.0 \%$ & $0.76 \%$ & $0.0 \%$ & & \\
\hline
\end{tabular}




\section{Discussion}

The role of the dentists in detecting oral cancerous and premalignant lesions is crucial. The main aim is to educate people about the importance of regular dental checkups and the relationship that many of the associated factors have with the development of oral premalignancy and malignancy (Goyal et al., 2012).

The current investigation is the first one to study the prevalence of potentially malignant lesions and oral cancer among smokers in Egypt, aiming to understand the current situation and relation between smoking habits and carcinogenesis.

We enrolled 1664 smokers of which $97.6 \%$ were males and $2.4 \%$ were females thus, gender correlation with lesions prevalence was difficult due to small number of female subjects.

All participants were found to consume tobacco in the form of cigarettes, shisha or both, with no subjects using pipes or cigars. Most smokers start the habit during their teens and early 20s. So, they are more likely to become heavy smokers by middle and old age (Kuper et al., 2002) in our study, the most prevalent age was $31-40$ years old reaching $25.3 \%$ of included individuals.

As tobacco use was found to increase the number of aneuploid nuclei in clinically healthy oral epithelium and thus, contribute to increased risk of the development of potentially malignant or malignant oral lesions (Souto et al., 2010). The current study recognized the impact of smoking both clinically and through histopathological evaluation of clinically suspicious oral lesions to detect dysplasia. Suspicious premalignant lesions in the study reached $6.4 \%$ of cases, including leukoplakia and oral lichen planus, while the malignant lesions hit $1.6 \%$ by OSCC and verrucous carcinoma.

Concerning OLP, $31.25 \%$ of patients consumed tobacco for 30 years and on histopathological examination two cases showed dysplasia. The prevalence of OLP which reached $2.88 \%$ among smokers in the present study was higher than previous studies; $1.4 \%$ in the work of Ali et al. (2013) (Kuwait), $1.7 \%$ by Chher et al. (2016) (Cambodia) and $2.5 \%$ by Patil et al. (2013) (India) with the majority of patients ranges from consuming cigarettes in the range of 1 to 2 packs per day.

Among leukoplakia patients, $44 \%$ consumed tobacco for 30 years and $45.7 \%$ smoked 2 packs per day. Leukoplakia represented $3.54 \%$ of the lesions, 10 cases out of 59 showed dysplasia. The leukoplakia results in the current study coincides with studies in Saudi Arabia (2.3\%), Iran (2.7\%) and Cambodia (3.5\%) (Al-Attas et al. 2014, Motamayel et al. 2013 and Chher et al. 2016), respectively. Other studies in India reported higher prevalence; 5.3\% (Priya et al, 2018), 8\% (Naveen-Kumar et al. 2016), 8.25\% (Patil et al., 2013), 14\% (Sujatha et al 2012) and in Yemen the prevalence was as high as $27 \%$ (Scheifele et al., 2007). Such higher values could be attributed to customs inherent to these populations related to smokeless tobacco preparations and highly irritant spices used besides tobacco smoking or deleterious smoking styles as reverse smoking habit.

In the current study, the commissures bilaterally were the most common mucosal site noted for leukoplakia whether homogenous or speckled, also some lesions presented at the floor of the mouth and tongue. The commissure along with the buccal mucosa were previously found to be the most common sites of occurrence of leukoplakia (Gaphor and Sabri 2014; Priya et al., 2018).

In the present study, $1.6 \%$ of the smoker population were diagnosed with malignant lesions (27 in number, 25 of which were OSCC and 2 were verrucous carcinoma). Previous studies showed almost the same prevalence of OSCC among different populations; in Yemen 1\% (Scheifele et al., 2007), in Iran 1.1\% (Motamayel et al, 2013), in Cambodia 2.2\% (Chher et al., 2016), while in India the percentage was higher and reached $4.09 \%$ (NaveenKumar et al., 2016). This is most probably due to the different forms of tobacco and smoking styles used in India and the spread of the habit in the Indian population.

A remark that can be of importance among patients with OSCC is that they were of low socioeconomic level, as shown by their level of education and residence with $40 \%$ of them smoking 2 packs of 
cigarettes per day and $32 \%$ consuming tobacco for 30 years. Unhealthy lifestyles, with nutritional deficiencies and poor hygienic habits, seems to promote the occurrence of such lesions in response tobacco use, and these factors were also reported in other studies (Shankar et al., 2010; Sujatha et al., 2012).

In general, the differences in prevalence of oral lesions among different populations could be also attributed to differences in the genetic make-up. Winn (2001) revealed that genetic factors play a role in the development of tobacco-related oral lesions. Genetic susceptibility to disease was found to be augmented by the epigenetic effects of environmental challenges, like tobacco smoking, along with the activities of non-coding miRNAs thus affecting carcinogenesis and behavior of oral lesions (Bergmeier, 2018).

Alcohol consumption is considered as a potential risk factor for oral cancer, but when it is consumed along with tobacco it increases the risk because of synergistic interaction (Ogden 2005 $\&$ Warnakulasuriya, 2009). That may be because alcohol increases the permeability of the oral mucous membranes, thereby increases the tissue penetration of tobacco smoke carcinogens and cause potentially malignant and malignant disorders (Kuper et al., 2002; Lee et al., 2003; Saraswathi et al., 2006; Cebeci et al., 2009 and Javed et al., 2010). However, and despite all previous evidence, none of the OSSC patients in the present study was found to consume alcohol besides smoking and only one patient with leukoplakia showing mild dysplasia did.

The results of the present study throw some light on the prevalence of potentially malignant lesions and oral cancer among smokers in the Egyptian population. Dentists must be aware of the risk of tobacco consumption on oral mucosa and the early signs of developing premalignant or malignant lesions and encourage the patient to quit. Tobacco cessation counseling should be part of the armamentarium of the dental office.

\section{Conflict of Interest}

The authors declare no conflict of interest.

\section{References}

Al-attas, S. A. et al. (2014) 'Prevalence of Potentially Malignant Oral Mucosal Lesions among Tobacco Users in Jeddah, Saudi Arabia', Asian Pac J Cancer Prev, 15(2), pp. 757-762. doi: http://dx.doi.org/10.7314/APJCP.2014.15.2.757.

Ali et al. (2013) 'Prevalence of oral mucosal lesions in patients of the Kuwait University Dental Center', The Saudi Dental Journal. King Saud University, 25(3), pp. 111-118. doi: 10.1016/j.sdentj.2013.05.003.

Al-mobeeriek, A. and Aldosari, A. M. (2009) 'Prevalence of oral lesions among Saudi dental patients', Ann Saudi Med, 29(5), pp. 365-368.

Aruna, D. S. et al. (2011) 'Retrospective study on risk habits among oral cancer patients in karnataka cancer therapy and research institute, hubli, India', Asian Pacific Journal of Cancer Prevention, 12(6), pp. 1561-1566.

Behura et al. (2015). Oral mucosal lesions associated with smokers and chewers-a case-control study in chennai population. Journal of clinical and diagnostic research: JCDR 9, ZC17.

Bergmeier, L. A. (2018) Oral Mucosa in Health and Disease. Edited by L. A. Bergmeier. London: Springer International Publishing AG. doi: https://doi.org/10.1007/978-3-319-56065-6.

Cebeci et al. (2009). Prevalence and distribution of oral mucosal lesions in an adult Turkish population. Med Oral Patol Oral Cir Bucal 14, E272277.

Chher et al. (2016). Prevalence of oral cancer, oral potentially malignant disorders and other oral mucosal lesions in Cambodia. Ethnicity \& health 23, $1-15$.

Day, B. W. N. and T. A. (2002) 'Oral Cancer and Precancerous Lesions', CA Cancer J Clin, 52(4), pp. 195-215.

Fouda, S. et al. (2018) 'Tobacco smoking in Egypt: A scoping literature review of its epidemiology and control measures', Eastern Mediterranean Health Journal, 24(2), pp. 198-215. doi: 10.26719/2018.24.2.198. 
Gaphor, S. M., and Sabri, Z. A. (2014). Prevalence of oral premalignant and malignant Lesions among referred Kurdish patients Attending Department of Oral and Maxilofasial in Sulaimani Teaching Hospital. IOSR Journal of Dental and Medical Sciences 13, 32-36.

Goyal, D. et al. (2013) 'An update on precancerous lesions of oral cavity', IJMDS, 2(1), pp. 70-75.

Jahanbani et al. (2009). Evaluation of oral mucosal lesions in 598 referred Iranian patients. The open dentistry journal 3,42 .

Javed et al. (2010). Oral mucosal disorders associated with habitual gutka usage: a review. Oral Surgery, Oral Medicine, Oral Pathology, Oral Radiology, and Endodontology 109, 857-864.

Kuper et al. (2002) "Tobacco use, cancer causation and public health impact". Journal of Internal Medicine.', Journal of Internal Medicine, 251(6), pp. 455-66. doi: doi:10.1046/j.13652796.2002.00993.x.

Lee et al. (2003). The precancer risk of betel quid chewing, tobacco use and alcohol consumption in oral leukoplakia and oral submucous fibrosis in southern Taiwan. British journal of cancer 88, 366.

Motamayel, F. A. et al. (2013) 'Prevalence of Oral Mucosal Lesions in Male Smokers and Nonsmokers', Chonnam Medical Journal, 49(2), pp. 65-68.

Naveen-Kumar et al. (2016). Various forms of tobacco usage and its associated oral mucosal lesions. Journal of clinical and experimental dentistry 8, e172.

Ogden, G. R. (2005). Alcohol and oral cancer. Alcohol 35, 169-173.

Patil et al. (2013). Prevalence of oral mucosal lesions in dental patients with tobacco smoking, chewing, and mixed habits: A cross-sectional study in South India. Journal of family \& community medicine 20, 130.

Pentenero et al. (2008). The prevalence of oral mucosal lesions in adults from the Turin area. Oral diseases 14, 356-366.

Priya et al. (2018). Evaluation of the prevalence of oral mucosal lesions in a population of eastern coast of South India. Journal of International Society of Preventive \& Community Dentistry 8, 396.

Rastogi et al. (2013). An insight to oral epithelial dysplasia. Int J Head Neck Surg 4, 74-82.

Saraswathi et al. (2006). Prevalence of oral lesions in relation to habits: Cross-sectional study in South India. Indian journal of dental research 17,121.

Scheifele et al. (2007). Prevalence of oral cancer and potentially malignant lesions among shammah users in Yemen. Oral oncology 43, 42-50.

Scully, C., and Felix, D. (2005). Oral medicineUpdate for the dental practitioner Aphthous and other common ulcers. British dental journal 199, 259.

Sham et al. (2003). The effects of tobacco use on oral health. Hong Kong Medical Journal.

Shankar et al. (2010). Health-Related Behaviors in Older Adults Relationships with Socioeconomic Status. American journal of preventive medicine. 38 . 39-46.

Souto, G. R. et al. (2010) 'Tobacco use increase the number of aneuploid nuclei in the clinically healthy oral epithelium', Oral Pathol Med, 39, pp. 605-610. doi: 10.1111/j.1600-0714.2010.00907.x.

Sujatha et al. (2012). Prevalence and correlation of oral lesions among tobacco smokers, tobacco chewers, areca nut and alcohol users. Asian Pacific Journal of Cancer Prevention 13, 1633-1637.

Taybos G. (2003). Oral Changes Associated with Tobacco Use. American Journal of the Medical Sciences, 326 (4), pp. 179-182.

Vellappally et al. (2007). Smoking related systemic and oral diseases. ACTA MEDICAHRADEC KRALOVE- 50, 161.

Warnakulasuriya et al. (2007). Oral cancer survival in young people in South East England. Oral oncology 43, 982-986.

Warnakulasuriya, S. (2009). Causes of oral cancer-an appraisal of controversies. British dental journal 207, 471

WHO (2013). Report on the global tobacco epidemic: enforcing bans on tobacco advertising, promotion and sponsorship, World Health Organization. 2013 
Winn, D. M. (2001) 'Tobacco use and oral 312. disease.', Journal of dental education, 65(4), pp. 306- 